\title{
Elemental diet plus glutamine for the prevention of mucositis in esophageal cancer patients receiving chemotherapy: a feasibility study
}

\author{
Yoshihiro Tanaka ${ }^{1}$ Takao Takahashi ${ }^{1} \cdot$ Kazuya Yamaguchi $^{1} \cdot$ Shinji Osada $^{1}$. \\ Toshio Shimokawa ${ }^{2} \cdot$ Kazuhiro Yoshida $^{1}$
}

Received: 9 December 2014 / Accepted: 27 July 2015 / Published online: 13 August 2015

(C) The Author(s) 2015. This article is published with open access at Springerlink.com

\begin{abstract}
Purpose We investigated the effect of glutamine (Gln) and an elemental diet (ED) on chemotherapy-induced oral mucositis in esophageal cancer patients.

Methods Thirty patients were randomized to the control group (no treatment: $n=10$ ), Gln group (oral intake of $8910 \mathrm{mg} \mathrm{Gln} /$ day: $n=10$ ), or Gln plus ED group (total oral intake of $8862 \mathrm{mg} \mathrm{Gln} /$ day, including the Gln in ED: $n=10$ ). Oral administration of Gln and ED began 1 week before chemotherapy and continued during treatment. Oral mucositis was evaluated during 2 cycles of chemotherapy using Common Terminology Criteria for Adverse Events v3.0.

Results The incidence of grade $\geq 2$ oral mucositis was $60 \%$ in the control group, $70 \%$ in the Gln group, and $10 \%$ in the Gln plus ED group. Gln plus ED showed a significant preventive effect on the development and severity of oral mucositis. By multivariate analysis, Gln plus ED and cancer stage were independent factors affecting chemotherapy-induced oral mucositis. The percentage of change in body weight and diamine oxidase activity from before chemotherapy was higher in the Gln plus ED group than in the control group.
\end{abstract}

Electronic supplementary material The online version of this article (doi:10.1007/s00520-015-2864-9) contains supplementary material, which is available to authorized users.

Yoshihiro Tanaka

yoshihirotana11@hotmail.com

1 Department of Surgical Oncology, Gifu Graduate School of Medicine, 1-1 Yanagido, Gifu City, Gifu 501-1194, Japan

2 Graduate School of Medicine and Engineering, University of Yamanashi, 4-4-37 Takeda, Kofu City 400-8510, Japan
Conclusions Oral administration of Gln plus ED may prevent chemotherapy-induced oral mucositis in esophageal cancer patients.

Keywords Esophageal cancer · Chemotherapy · Mucositis · Glutamine $\cdot$ Elemental diet

\section{Introduction}

Although morbidity and mortality rates after surgical treatment for advanced esophageal cancer have decreased, the 5year survival rate after curative surgery is still only $20-36 \%$ [1]. In patients with operable esophageal cancer, there is evidence supporting the use of preoperative chemotherapy or preoperative chemoradiation [2-4]. Patients with unresectable or inoperable esophageal disease have also been treated with various chemotherapy strategies [5]. Chemotherapy can significantly improve the clinical outcomes of cancer patients, but it can also result in serious adverse effects [6]. In esophageal cancer, treatment with 5-fluorouracil (5-FU)/cisplatin plus docetaxel (DCF) is reported to be associated with increased response rates but also with an increased incidence of toxicities, the most common of which are hematological and gastrointestinal (GI) [7, 8]. Triplet regimen had high response rate and also was associated with higher rates of grade of stomatitis [9]. GI toxicities caused by chemotherapy can negatively affect patients' nutritional status by decreasing food intake and can result in a delay or discontinuation of chemotherapy [10], thereby negatively affecting tumor response to treatment; in addition, GI toxicities could lead to a delay in elective surgery and increase the risk of operative complications. Oral mucositis, one of the most common GI toxicities, results in increased pain, difficulty in swallowing, nutritional compromise, and an increased risk of infection. 
Nishimura et al. [11] reported that the incidence of oral mucositis (Common Terminology Criteria for Adverse Events [CTCAE] [12] grade $\geq 1$ ) was the highest during chemotherapy for breast cancer $(76.5 \%)$, followed by chemotherapy for head and neck cancer $(67.7 \%)$, colorectal cancer $(63 \%)$, esophageal cancer $(57.8 \%)$, and malignant lymphoma $(42.9 \%)$. Several reports indicated that the glutamine (Gln) decreased only the subjective symptoms of stomatitis [13-15]. Even if there was objective effect, a large amount of Gln was administered $[16,17]$. It was difficult to formulate a large amount of Gln in the practical treatment. Recently, efficacy of elemental diet (including Gln) for chemotherapy-induced oral mucositis in esophageal cancer (grade 1 stomatitis) and colorectal cancer (grade 1-3 stomatitis) patients was reported, and their concept of treatment were after experience of stomatitis $[18,19]$. In our pilot study, oral Gln and elemental diet (ED) which was administered from before starting of chemotherapy was found to help maintain patients' nutritional status and to reduce the incidence of oral mucositis in esophageal cancer (unpublished data).

We therefore conducted a randomized phase II study for investigation of efficacy and safety at Gln plus ED or Gln alone compared to no treatment on oral mucositis in patients with esophageal cancer undergoing chemotherapy.

In addition, because oral mucositis is reported to be caused by chemotherapy-induced mucosal damage or infection and inflammation by indigenous oral bacteria [20], we also measured and compared the mucosal integrity and degree of inflammation between the treatment groups.

\section{Methods}

\section{Ethical considerations}

This trial was conducted in accordance with the World Medical Association Declaration of Helsinki and registered with the University Hospital Medical Information Network Clinical Trials Registry (UMIN000008338). The study protocol was approved by the Ethics Committee of Gifu University Hospital, and written informed consent was obtained from all patients.

\section{Eligibility criteria}

To be eligible for the study, patients had to be at least 18 years of age at the time of registration and have histologically or cytologically confirmed esophageal squamous cell carcinoma or adenocarcinoma.

Disease staging was defined according to the Japanese Society for Esophageal Disease guidelines (10th edition) [21]. Patients also had to have an Eastern Cooperative Oncology Group performance status of 0-1 [22], a life expectancy of
$>12$ weeks, and adequate liver, bone marrow, renal, and cardiovascular function (serum bilirubin $\leq 1.5 \mathrm{mg} / \mathrm{dL}$, neutrophil count $\geq 1500 / \mathrm{mm}^{3}$, serum aspartate aminotransferase and alanine aminotransferase levels $\leq$ twice the upper limit of normal range, platelet count $\geq 10 \times 104 / \mathrm{mm}^{3}$, hemoglobin $\geq 8.0 \mathrm{~g} / \mathrm{dL}$, and creatinine $\leq 1.2 \mathrm{mg} / \mathrm{dL}$ [or creatinine clearance $>60 \mathrm{~mL} /$ $\min ]$ ).

Patients previously treated with chemotherapy for malignant disease or irradiation to major bone areas were excluded from the study. The major exclusion criteria included serious concomitant illness, symptomatic infectious disease, severe drug allergy, symptomatic peripheral neuropathy, or uncontrolled diabetes mellitus.

\section{Study drug}

In the Gln group, patients received $8910 \mathrm{mg}$ of Gln daily. In the Gln plus ED group, patients received $6930 \mathrm{mg}$ of Gln and one pack of Elental ${ }^{\circledR}(300 \mathrm{~mL} /$ day [300 kcal/day]; Supplementary Table S1) daily, based on a previous study [1]. In the Gln plus ED group, the amount of total Gln taken daily was equal to that in the Gln group. In all patients, calorie intake was restricted to $30 \mathrm{kcal} \mathrm{kg}^{-1} \mathrm{day}^{-1}$.

Patients received either DCF or docetaxel/nedaplatin $/ \mathrm{S}-1$ (DGS) [7, 23] chemotherapy regimens.

Patients undergoing DCF chemotherapy were administered $35 \mathrm{mg} / \mathrm{m}^{2}$ of docetaxel and $40 \mathrm{mg} / \mathrm{m}^{2}$ of cisplatin on days 1 and 15 and $400 \mathrm{mg} / \mathrm{m}^{2}$ of 5 -FU on days $1-5$ and $15-19$ of every 28-day cycle. Patients undergoing DGS chemotherapy were administered $35 \mathrm{mg} / \mathrm{m}^{2}$ of docetaxel and $40 \mathrm{mg} / \mathrm{m}^{2}$ of nedaplatin on day 7 and $80 \mathrm{mg} / \mathrm{m}^{2}$ of S-1 on days $1-14$ of every 28-day cycle. Except for patients with stage IVb disease, all patients underwent surgery.

\section{Study design}

This study is a randomized phase II study for investigation of efficacy and safety at Gln + ED treatment. We could estimate the effect size for each arm. We will plan a multicenter randomized phase III study using these estimated effect sizes for calculation of sample size. The primary objective of this study was to determine the effect of Gln and Gln plus ED on the incidence grade of oral mucositis [9] during 2 cycles of chemotherapy. After confirmation of eligibility, patients were randomized to the chemotherapy-only group (control), Gln group (Gln) (marzulene-S ${ }^{\circledR}$; Ajinomoto Pharmaceuticals Co., Ltd.), or Gln plus ED (Elental ${ }^{\circledR}$; Ajinomoto Pharmaceuticals Co., Ltd) group (Gln plus ED). In the Gln and Gln plus ED groups, each drug was administered orally beginning 1 week before chemotherapy and was continued between rounds of chemotherapy. Patients were assigned in a 1:1:1 ratio to receive Gln, Gln plus ED, or no additional treatment (control). During the chemotherapy cycles, patients continued to receive Gln or Gln 
plus ED every day. Total daily calories consumed were restricted to $30 \mathrm{kcal} /$ body weight in all patients, and the amount of food intake was directly reported by patients. In cases where food consumption was $70 \%$ or less of the defined amount for 3 days, the patient was withdrawn from the study because of the significant difference in calorie intake. Oral mucositis was assessed by two trained physicians who were blinded whether administered or not. The severity grade was assessed before chemotherapy, after the first cycle of chemotherapy, and after the second cycle of chemotherapy. Before chemotherapy, a dentist has performed oral care for dental caries and periodontal disease to all the patients. Patient education in oral selfcare was performed by nurses, too. We also measured weight; plasma diamine oxidase (DAO) activity, which is a reliable indicator of intestinal mucosal integrity [24]; C-reactive protein (CRP) level, which was used as an inflammatory indicator; turnover rate of plasma proteins (prealbumin, transferrin, and retinol binding protein [RBP]), as indicators of nutritional status; and plasma concentration of various amino acids such as Gln and histidine. We assessed tumor response according to the Response Evaluation Criteria in Solid Tumors guidelines [25] after 2 cycles of chemotherapy. A barium meal study, endoscopy, ultrasonography, and computed tomography or magnetic resonance imaging were used to evaluate the response status of measurable lesions. We defined complete response as complete disappearance of all clinically detectable malignant disease and partial response as a $>30 \%$ decrease in the sum of the perpendicular diameters of all measurable lesions present for at least 4 weeks. We defined progressive disease as either a $>20 \%$ increase in the sum of the products of measurable lesions over the smallest sum observed or the appearance of new lesions. Stable disease did not qualify as complete response, partial response, or progressive disease. We investigated the incidence of any complications at the time of operation.

\section{Other adverse events}

Side effect was assessed using the National Cancer Institute CTCAE version 3.0 [12].

\section{Statistical analysis}

The primary endpoint was the pairwise comparison of incidence grade of mucositis between each of the two experimental arms (Gln group and Gln plus ED group) and the control arm. Secondary endpoints included a comparison of patient demographics and confirmation of laboratory changes. For the primary endpoint, the Mann-Whitney $U$ test with Bonferroni correction was used to assess the reduction of mucositis incidence. A proportional odds model was used to the evaluation of treatment effect (Gln and Gln + ED) and adjusted effect of five covariates (age, sex, cancer stage, treatment, and body mass index). The estimated model may have multicollinearity, so we applied backward stepwise method using Akaike's information criteria as variable selection measure. Secondary endpoints were assessed using Dunnett's multiple comparison method. Statistical analyses were performed using $\mathrm{R}$ version 3.1.1. A $P$ value $<0.05$ was considered to be statistically significant.

\section{Results}

\section{Patients}

From June 2010 to March 2013, 30 patients were enrolled and randomized to either the Gln plus ED group $(n=10)$, Gln group $(n=10)$, or control group $(n=10)$. The baseline characteristics of the patients are shown in Table 1. All included patients consumed more than $70 \%$ of the established calorie requirements. With respect to the amount of anti-cancer drugs that was actually administered, all 30 patients received full doses in the first cycle; in the second cycle, 2 patients in the control group, 1 patient in the Gln group, and 2 patients in the ED plus Gln group had a $10 \%$ dose reduction because of hematological adverse events.

\section{Efficacy}

\section{Oral mucositis}

During this study, oral mucositis of grade $\geq 2$ developed in approximately $60 \%$ of patients in the control group, and the incidence of oral mucositis was significantly lower in the Gln plus ED group (10 \%) than in the control group (Fig. 1a). In addition, during the first cycle of chemotherapy, the incidence of oral mucositis was significantly lower in the Gln plus ED group than in the control group $(P=0.040)$ (Fig. 1b). However, no significant difference between the control and Gln plus ED groups was observed during the second cycle of chemotherapy (Fig. 1c). On the other hand, no significant difference between the control and Gln groups was observed during this study (Fig. 1a, b, c). The results of the multivariate analysis demonstrated that in addition to Gln plus ED (odds ratio $=0.1, P=0.02$ ), cancer stage (odds ratio $=13.3$, $P=0.01$ ) was an independent factor affecting mucositis grade during chemotherapy (Table 2).

\section{Laboratory tests}

No significant differences in the absolute values of laboratory tests before chemotherapy, except for plasma DAO (5.81 in the control group, 6.63 in the Gln group, and 3.43 in the Gln plus ED group, respectively), were observed among the groups. The Gln plus ED group showed a significant increase in DAO activity during chemotherapy (approximately 1.3 
Table 1 Characteristics of patients

\begin{tabular}{|c|c|c|c|c|}
\hline Group & & Control $(n=10)$ & $\mathrm{G} \ln (n=10)$ & Gln plus ED $(n=10)$ \\
\hline \multirow[t]{2}{*}{ Regimen } & DCF & 4 & 4 & 5 \\
\hline & DGS & 6 & 6 & 5 \\
\hline \multirow[t]{2}{*}{ Daily calorie intake including ED (kcal) } & & 2100 & 2100 & 2100 \\
\hline & & $(1800-2100)$ & $(1600-2100)$ & $(2100-2100)$ \\
\hline \multirow[t]{2}{*}{ Age (years) } & & 68 & 73.5 & 75 \\
\hline & & $49-82$ & $68-78$ & $58-83$ \\
\hline \multirow[t]{2}{*}{ Sex } & Female & 1 & 3 & 0 \\
\hline & Male & 9 & 7 & 10 \\
\hline Cancer site & & Ce 1, Lt 3, Mt 6 & Ae 1, Lt 2, Mt 4, Mt-Ut 2, Ut 1 & Lt 2, Mt 8 \\
\hline \multirow[t]{2}{*}{ Radiation } & Yes & 0 & 0 & 0 \\
\hline & No & 10 & 10 & 10 \\
\hline \multirow[t]{4}{*}{ Stage } & II & 2 & 4 & 1 \\
\hline & III & 4 & 1 & 5 \\
\hline & IVa & 3 & 3 & 0 \\
\hline & $\mathrm{IVb}$ & 1 & 2 & 4 \\
\hline BMI & & $21.75(18.37-25.59)$ & $22.42(16.60-25.20)$ & $21.08(14.60-24.20)$ \\
\hline
\end{tabular}

Ae abdominal esophageal site, $B M I$ body mass index, $C e$ cervical esophageal site, $D C F$ docetaxel + cisplatin +5 -fluorouracil, $D G S$ docetaxel + nedaplatin + S-1, ED elemental diet, Gln glutamine, $L t$ lower thoracic esophageal site, $M t$ middle thoracic esophageal site, $U t$ upper thoracic esophageal site

times the DAO activity measured before chemotherapy) (Table 3). In the control group, there was significant weight loss during chemotherapy $(\Delta$ mean $=-5.4 \%, P=0.01)$. On the other hand, body weight was maintained in the Gln plus ED group, and changes in body weight were significantly different between the Gln plus ED and control groups (1.70 vs. $-5.40 \%$, respectively; $P=0.01$ ) (Table 3 ). T helper 1 cells and immunoglobulin A levels were significantly reduced during chemotherapy ( -27.63 and $-25.90 \%$, respectively). However, there was no significant difference in the rate of change of these values observed among the groups (Table 3).

During chemotherapy, there were no significant differences in other parameters, such as CRP and rapid turnover proteins (prealbumin, transferrin, RBP), which are known to reflect systemic inflammatory status and nutritional index. After receiving chemotherapy, the plasma concentrations of various amino acids were measured, and the plasma concentrations of some amino acids tended to be lower in the control group than in the other groups. For example, plasma Gln tended to be higher in the Gln and Gln plus ED groups than in the control group (-3.64, 9.91, and 9.59\%, respectively; Table 3).

\section{Response rate}

The treatment did not negatively affect patients' response rates to chemotherapy (Table 4). In the Gln plus ED group, the response rate was $90 \%$ (complete response, $10 \%$; partial response, $80 \%$; stable disease, $10 \%$ ). In the control and Gln groups, the response rates were 70 and $80 \%$, respectively (control group: complete response $10 \%$, partial response $60 \%$, stable disease $20 \%$, progressive disease $10 \%$; Gln group: complete response $30 \%$, partial response $50 \%$, stable disease $20 \%$ ).

\section{Adverse events and operative complications}

Detailed information on the adverse events reported in the control, Gln, and Gln plus ED groups is presented in Table 5. In all groups, the most common grade $3 / 4$ hematological toxicity was neutropenia.

Non-hematological toxicities other than oral mucositis were mild in all groups (including grade 2 diarrhea, anorexia, and alopecia). In one case in the Gln group and two cases in the control group, the second cycle of chemotherapy was delayed for 3-4 days because of grade 3 mucositis. Surgical resection was performed in seven cases in the Gln plus ED group, in eight cases in the Gln group, and in nine cases in the control group. Postoperative complications were not observed in any group, and administration of ED did not interfere with any planned radical surgeries.

\section{Discussion}

This is the first study to show that Gln plus ED can prevent oral mucositis in patients receiving chemotherapy. Moreover, the results of the multivariate analysis demonstrated that Gln plus ED and cancer stage were independent factors affecting 

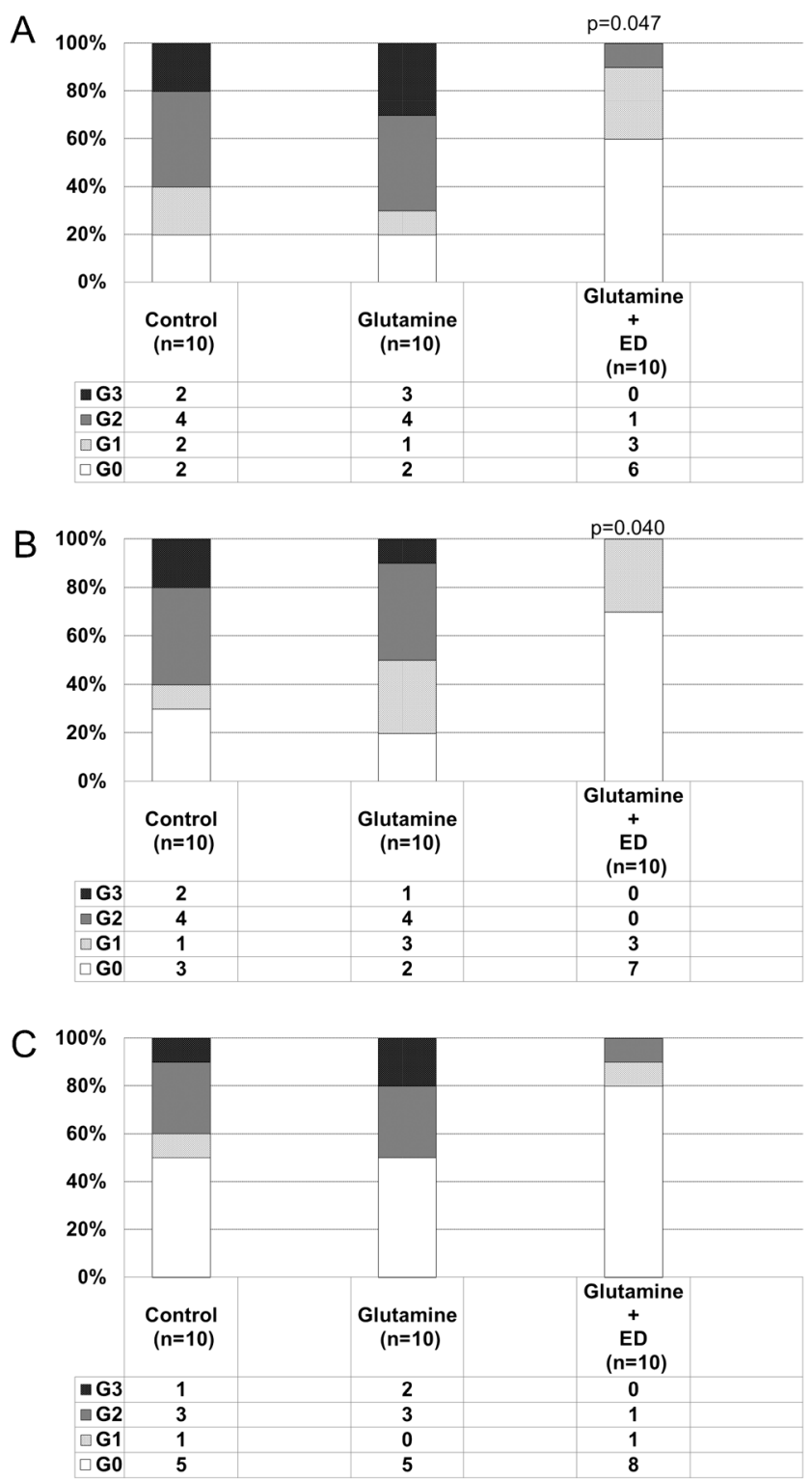

Fig. 1 Incidence of oral mucositis during chemotherapy (\%). a During 2 cycles of chemotherapy. b During the first cycle of chemotherapy. c During the second cycle of chemotherapy. $E D$ elemental diet, $G$ grade, $n s$ not significant

mucositis grade during chemotherapy. Additionally, in our study, Gln plus ED had beneficial effects on the integrity of the intestinal mucosa, as indicated by DAO measurements and body weight. In the Gln plus ED group, there was a significant difference during chemotherapy in the inhibitory effect against stomatitis. But in cycle 2, there was no significance of prevention. Probably because of the dose reduction in five cases in cycle 2 and also small sample size, significant difference was not seen in cycle 2 . When enteral nutrients are administered, poor compliance becomes a serious problem. However, in this study, all patients completed the scheduled treatment with $\mathrm{ED}$, which suggested good patient compliance. One of the reasons why the high intake ratio was maintained may be the choices offered to patients on how to take ED, such as adding flavoring agents or a jelly mix. Previous reports indicated that the Gln decreased the subjective symptoms of stomatitis in chemotherapy, radiotherapy, and hematopoietic stem cell transplantation [13-15]. Our study showed that Gln alone did not have a preventive effect against oral mucositis, in spite of the same Gln content as Gln plus ED. This was in contrast to previous studies that reported that oral Gln reduced the incidence and severity of mucositis [13-17]. In our trial, marzulene ${ }^{\circledR}$ (which is largely composed of Gln) was administered at a dose of $8910 \mathrm{mg} /$ day as an oral Gln formulation. Previous reports administered Gln doses of 30 and $24 \mathrm{~g}$ /day $[16,17]$, respectively; thus, the dose used in this study may be inadequate to prevent oral mucositis. Saforis ${ }^{\mathrm{TM}}$ (Eisai Co., Ltd.), which is composed of Gln in a novel, proprietary, drug delivery system, is used for preventing oral mucositis. Peterson et al. [26] reported that Saforis ${ }^{\mathrm{TM}}$ significantly prevented oral mucositis in cancer patients receiving chemotherapy when administered at a dose of $2.5 \mathrm{~g}$, three times a day, for 14 days. Comparing the local concentration of marzulene ${ }^{\circledR}$ with that of Saforis ${ }^{\mathrm{TM}}$ is difficult, but Saforis ${ }^{\mathrm{TM}}$ might have a higher concentration than marzulene ${ }^{\circledR}$ in epithelial oral mucosal cells because of its ability to facilitate the uptake of Gln by more than 100-fold. May et al. suggested that the mixture of hydroxyl-methylbutyrate, arginine, and Gln (HMB/Arg/Gln $16 \mathrm{~g}$ ) was effective against cancer cachexia [27]. Additionally, this paper suggested that one of the mechanisms of this effect was that Gln is a substrate for enterocytes and lymphocytes, acting to improve nitrogen retention, lower the incidence of infections, and decrease tumor growth and chemotherapy toxicity. However, this mixture has a higher Gln content than the dose used in this study. Therefore, the Gln dose in this study might be inadequate to prevent oral mucositis. Gln plus ED prevented oral mucositis in patients receiving chemotherapy, despite the fact that this regimen included the same amount of Gln as marzulene ${ }^{\circledR}$ alone. It cannot be ruled out that absorptive kinetics may be different between marzulene ${ }^{\circledR}$ and marzulene ${ }^{\circledR}$ plus ED, but various amino acids, including Gln, in ED might have resulted in the prevention of oral mucositis. Supportively, efficacy of ED for chemotherapyinduced oral mucositis in esophageal cancer and colorectal cancer patients was recently reported, but their concept of treatment was after experience of stomatitis $[18,19]$.

Although the pathogenesis of mucositis remains to be completely elucidated, it has been reported that chemotherapy damages DNA through production of reactive oxygen species, induces apoptosis by upregulating the expression of intracellular molecules such as NF-kB, and produces several cytokines such as interleukin-1 beta, interleukin-6 (IL-6), and tumor necrosis factor-alpha (TNF-alpha) (direct mechanism) [20]. The protective effect of ED on mucosal tissue in Crohn's disease has been widely reported [28, 29]. In clinical practice, ED has a clear suppressive effect on clinical and endoscopic 
Table 2 Multivariate analysis for independent factors affecting increased mucositis grade during chemotherapy

\begin{tabular}{|c|c|c|c|c|c|c|}
\hline Factor & & Number of patients & Odds ratio $(95 \% \mathrm{CI})$ & $P$ value & Odds ratio $(95 \% \mathrm{CI})$ & $P$ value \\
\hline \multirow[t]{2}{*}{ Gln } & With & 20 & $4.1(0.6-33.7)$ & 0.17 & $4.0(0.7-29.6)$ & 0.15 \\
\hline & Without & 10 & & & & \\
\hline \multirow[t]{2}{*}{ Gln plus ED } & With & 20 & $0.1(0.0-0.7)$ & $0.03^{*}$ & $0.1(0.0-0.6)$ & $0.02 *$ \\
\hline & Without & 10 & & & & \\
\hline \multirow[t]{2}{*}{ Age, years } & $<70$ & 16 & $3.1(0.7-16.5)$ & 0.16 & $3.1(0.7-16.1)$ & 0.15 \\
\hline & $\geq 70$ & 14 & & & & \\
\hline \multirow[t]{2}{*}{ Sex } & Male & 26 & $0.9(0.1-7.7)$ & 0.95 & - & - \\
\hline & Female & 4 & & & & \\
\hline \multirow[t]{2}{*}{ Cancer stage } & IVa,b & 13 & $14.6(1.9-150.0)$ & $0.01 *$ & $13.3(1.9-123.2)$ & $0.01^{*}$ \\
\hline & II-III & 17 & & & & \\
\hline \multirow[t]{2}{*}{ Treatment } & First line & 7 & $0.2(0.0-2.1)$ & 0.19 & $0.2(0.0-1.6)$ & 0.13 \\
\hline & NAC & 23 & & & & \\
\hline \multirow[t]{2}{*}{ BMI } & $<21$ & 13 & $1.9(0.4-9.6)$ & 0.44 & - & - \\
\hline & $\geq 21$ & 17 & & & & \\
\hline
\end{tabular}

$B M I$ body mass index, $E D$ elemental diet, Gln glutamine, $N A C$ neoadjuvant chemotherapy

$* p<0.05$

disease activities and the levels of mucosal inflammatory cytokines, such as interleukin-1 beta, IL-6, and TNF-alpha [28].

Moreover, histidine, one of the constituent amino acids of ED, inhibited lipopolysaccharide-induced TNF-alpha and IL-6 production by mouse macrophages in a concentrationdependent manner [29]. Therefore, in chemotherapy-induced

Table 3 Percentage changes in laboratory test results after the first and second cycles of chemotherapy compared to pre-chemotherapy

\begin{tabular}{|c|c|c|c|c|c|c|c|c|c|c|}
\hline & \multicolumn{5}{|c|}{ Post first cycle } & \multicolumn{5}{|c|}{ Post second cycle } \\
\hline & \multicolumn{3}{|c|}{$\Delta$ mean $(\%)$} & \multicolumn{2}{|c|}{$P$ value } & \multicolumn{3}{|c|}{$\Delta$ mean $(\%)$} & \multicolumn{2}{|c|}{$P$ value } \\
\hline & Control & Gln & Gln plus ED & Gln & Gln plus ED & Control & Gln & Gln plus ED & Gln & Gln plus ED \\
\hline Weight & -3.54 & -2.9 & -0.38 & 0.89 & 0.11 & -5.40 & -3.9 & 1.70 & 0.73 & $0.01 *$ \\
\hline Prealbumin & 20.59 & 2.61 & 8.79 & 0.59 & 0.79 & 16.74 & 7.89 & 21.34 & 0.82 & 0.95 \\
\hline RBP & 33.54 & 32.61 & 19.75 & 1.00 & 0.91 & 32.47 & 27.66 & 26.25 & 0.96 & 0.94 \\
\hline Transferrin & -6.39 & -18.35 & -6.44 & 0.34 & 1.00 & -8.48 & -15.94 & -2.38 & 0.67 & 0.76 \\
\hline Ferritin & 102.93 & 186.13 & 183.79 & 0.41 & 0.43 & 283.18 & 246.26 & 383.87 & 0.98 & 0.85 \\
\hline Lymphocyte counts & -22.41 & -27.82 & -23.99 & 0.91 & 0.99 & -14.37 & -21.94 & 4.59 & 0.83 & 0.34 \\
\hline DAO & -6.81 & -25.26 & 33.7 & 0.46 & $0.04 *$ & -10.13 & -27.17 & 26.68 & 0.56 & 0.10 \\
\hline HLA-DR & -4.35 & -11.52 & -11.04 & 0.72 & 0.75 & -1.61 & -2.84 & -4.04 & 0.99 & 0.97 \\
\hline Th1 & -20.79 & -20.52 & -17.06 & 1.00 & 0.86 & -27.63 & -26.08 & -26.69 & 0.96 & 0.98 \\
\hline Th2 & 39.95 & -7.18 & -18.26 & 0.45 & 0.31 & 15.64 & -3.97 & -37.48 & 0.74 & 0.15 \\
\hline Th1/Th2 & -2.51 & 1.9 & 30.14 & 0.98 & 0.38 & -5.72 & 10.94 & 58.06 & 0.90 & 0.25 \\
\hline CD4 & 61.66 & 22.77 & 28.1 & 0.20 & 0.29 & 52.35 & 26.57 & -0.05 & 0.46 & 0.07 \\
\hline CD8 & -19.94 & -14.38 & -15.5 & 0.84 & 0.90 & -15.35 & -14.18 & -2.77 & 0.99 & 0.28 \\
\hline CD4/CD8 ratio & 139.99 & 103.77 & 62.91 & 0.87 & 0.54 & 96.77 & 97.47 & 8.99 & 1.00 & 0.37 \\
\hline CRP & 328.85 & 1508.77 & 268.03 & 0.17 & 0.99 & 842.93 & 1592.76 & 68.73 & 0.55 & 0.53 \\
\hline Albumin & -2.73 & -10.17 & -8.27 & 0.19 & 0.37 & -4.21 & -12.14 & -4.24 & 0.16 & 1.00 \\
\hline $\operatorname{IgA}$ & -22.42 & -16.09 & -22.73 & 0.51 & 1.00 & -25.90 & -23.75 & -24.1 & 0.960 & 0.97 \\
\hline Glutamine & -13.64 & 2.9 & 0.87 & 0.23 & 0.31 & -3.64 & 9.91 & 9.59 & 0.20 & 0.21 \\
\hline Histidine & -14.98 & -4.50 & -0.29 & 0.59 & 0.37 & -13.67 & 2.22 & 0.03 & 0.16 & 0.25 \\
\hline
\end{tabular}

$C R P$ C-reactive protein, $D A O$ diamine oxidase, $E D$ elemental diet, $G l n$ glutamine, $I g A$ immunoglobulin $\mathrm{A}, R B P$ retinol binding protein, $T h \mathrm{~T}$ helper cell $* p<0.05$ 
Table 4 Chemotherapy response rates in each group

\begin{tabular}{lllll}
\hline & CR & PR & SD & PD \\
\hline Control & 1 & 6 & 2 & 1 \\
& $(10)$ & $(60)$ & $(20)$ & $(10)$ \\
Gln & 3 & 5 & 2 & 0 \\
& $(30)$ & $(50)$ & $(20)$ & $(0)$ \\
Gln + ED & 1 & 8 & 1 & 0 \\
& $(10)$ & $(80)$ & $(10)$ & $(0)$ \\
\hline
\end{tabular}

Data are presented as the number of patients. Percentage are noted in parentheses $C R$ complete response, $P R$ partial response, $S D$ stable disease, $P D$ progressive disease, $G l n$ glutamine, $E D$ elemental diet

mucositis, it has been hypothesized that ED might also have a mucosal protective effect and prevent oral mucositis. In fact, our trial showed significantly higher levels of DAO activity, an indicator of intestinal mucosal integrity, in the Gln plus ED group than in the control group. DAO activity was previously reported to be an index of intestinal mucosal integrity. In humans and rodents, DAO is detected in various tissues, including the small intestine and kidney, but may also be found in the plasma [30]. Plasma DAO activity correlated with DAO activity in the villi of small intestinal mucosa in rats [30] and also correlated with the severity of small intestinal mucosal lesions induced by anti-cancer drugs [31, 32]. Recently, measuring DAO activity in gastric cancer patients was reported to be useful not only as an indicator of mucosal injury but also for the evaluation of coinciding GI tract toxicities induced by anti-cancer drugs, such as 5-FU [31].

Therefore, the effect of ED on mucositis might result from maintaining mucosal integrity, indicated by higher DAO activity. In this study, from the results of the multivariate analysis, cancer stage IV was an independent influential factor

affecting increased mucositis grade during chemotherapy. Accordingly, previous reports have demonstrated increased levels of plasma cytokines, such as interleukin-1 beta, IL-6, and TNF-alpha in patients with advanced cancer stages. Although daily calorie intake was restricted to the same amount among the three groups in our trial, body weight was significantly decreased in the control group, and body weight in the Gln plus ED group was maintained after chemotherapy. The maintenance of body weight in the Gln plus ED group may be explained by two possible mechanisms. First, ED and Gln may have maintained mucosal integrity not only in the oral cavity but also in the GI tract, thereby resulting in maintained nutrient absorption. Second, ED included an amino acid formulation and not protein. Patients may be unable to digest food because of chemotherapy-induced GI toxicities. In this situation, amino acids may be more suitable than proteins to provide nutrients, as proteins require digestion. Levels of rapid turnover proteins, known to reflect nutritional index, were not dependent on body weight loss in the control group. Previously, Georgiannos et al. suggested that patients who lost weight $(>7.5 \%)$ had higher plasma CRP levels and lower prealbumin levels $(P<0.05)$ compared to both weight-stable cancer patients and controls [33]. In our trial, mean body weight loss was $5.4 \%$ in the control group, and lower than $7.5 \%$. Moreover, no significant increase in CRP levels during chemotherapy was observed in the control group. Therefore, rapid turnover proteins such as prealbumin were not significantly decreased in the control group in our study.

It was recently reported that enteral administration of amino acid mixtures enabled normal amino acid absorption in patients with mucositis [34]. On the other hand, it was also reported that absorption of long-chain fatty acids might have been reduced in patients with GI mucositis [35]. This also suggested that ED, which is composed of amino acids (not

Table 5 Adverse events according to treatment group

\begin{tabular}{|c|c|c|c|c|c|c|c|c|c|c|c|c|c|}
\hline & & \multicolumn{4}{|c|}{ Control } & \multicolumn{4}{|l|}{ Gln } & \multicolumn{4}{|c|}{ Gln plus ED } \\
\hline & & $\begin{array}{l}\text { Grade } \\
1\end{array}$ & $\begin{array}{l}\text { Grade } \\
2\end{array}$ & $\begin{array}{l}\text { Grade } \\
3\end{array}$ & $\begin{array}{l}\text { Grade } \\
4\end{array}$ & $\begin{array}{l}\text { Grade } \\
1\end{array}$ & $\begin{array}{l}\text { Grade } \\
2\end{array}$ & $\begin{array}{l}\text { Grade } \\
3\end{array}$ & $\begin{array}{l}\text { Grade } \\
4\end{array}$ & $\begin{array}{l}\text { Grade } \\
1\end{array}$ & $\begin{array}{l}\text { Grade } \\
2\end{array}$ & $\begin{array}{l}\text { Grade } \\
3\end{array}$ & $\begin{array}{l}\text { Grade } \\
4\end{array}$ \\
\hline \multirow[t]{3}{*}{ Hematological adverse events } & Leukopenia & & 1 & & & & 2 & & & & & & 1 \\
\hline & Neutropenia & & & 4 & & & & 3 & & & & 2 & \\
\hline & Anemia & & & & & & & & & & 1 & & \\
\hline \multirow{7}{*}{$\begin{array}{l}\text { Non-hematological adverse } \\
\text { events }\end{array}$} & Diarrhea & & 4 & & & & 4 & & & 1 & 4 & & \\
\hline & Anorexia & & 4 & & & 2 & 2 & & & & 3 & & \\
\hline & Dysgeusia & 1 & 2 & & & & 1 & & & & & & \\
\hline & Alopecia & 2 & 7 & & & 3 & 7 & & & & 7 & & \\
\hline & Nausea & & & & & & & & & & 1 & & \\
\hline & Edema & & & & & & & & & & 1 & & \\
\hline & Malaise & & & & & & & & & & 1 & & \\
\hline
\end{tabular}

ED elemental diet, Gln glutamine 
including protein) and a small quantity of fatty acids, might be the best source of enteral nutrients during cancer chemotherapy. In our trial, we measured plasma concentrations of various amino acids. However, no significant differences in the concentrations of plasma Gln or other amino acids were observed either before, between, or after the administration of ED and/or Gln in any groups. This may be due to the fact that Gln and other amino acids are almost locally consumed as a source of energy or anti-inflammatory effects in the gastrointestinal tract.

The above mechanisms of action of Gln plus ED on oral mucositis are completely speculative. Therefore, further experimental studies are needed to confirm the mechanism of the action of ED and/or Gln on mucositis. Moreover, one of the limitations of our study was the small sample size studied at a single site. Hence, we are currently conducting a large, multicenter study to confirm the beneficial effects of ED on chemotherapy-induced oral mucositis in esophageal cancer patients.

In conclusion, Gln plus ED may prevent chemotherapyinduced oral mucositis in esophageal cancer patients, and this effect may contribute to the maintenance of intestinal mucosal integrity and body weight during chemotherapy. These results would suggest that this will be tempered with recommendation for a multicenter RCT.

Acknowledgments We would like to thank Editage for providing editorial assistance.

Conflict of interest The authors declare that they have no competing interests.

Open Access This article is distributed under the terms of the Creative Commons Attribution-NonCommercial 4.0 International License (http://creativecommons.org/licenses/by-nc/4.0/), which permits any noncommercial use, distribution, and reproduction in any medium, provided you give appropriate credit to the original author(s) and the source, provide a link to the Creative Commons license, and indicate if changes were made.

\section{References}

1. Bagheri R, Maddah G, Saedi HS, Sadeghian MH, Roodbari S (2011) Bone marrow involvement in esophageal cancer patients who underwent surgical resection. Eur J Cardiothorac Surg 40(2): 343-346

2. Ando N (2011) Progress in multidisciplinary treatment for esophageal cancer in Japan as reflected in JCOG studies. Esophagus 8: 151-157

3. Khaldoun A, Jonathan RS, et al. (2012) Multimodality approach for locally advanced esophageal cancer. World J Gastroenterol 18(40): 5679-5687

4. Allum WH, Stenning SP, Bancewicz J, Clark PI, Langley RE, et al (2009) Long-term results of a randomized trial of surgery with or without preoperative chemotherapy in esophageal cancer. J Clin Oncol 27:5062-5067

5. Osaka Y, Shinohara M, Hoshino S, et al (2011) Phase II study of combined chemotherapy with docetaxel, CDDP and 5-Fu for highly advanced esophageal cancer. Anticancer Res 31:633-638

6. Mauer AM, Kraut EH, Krauss SA, Ansari RH, et al. (2005) Phase II trial of oxaliplatin, leucovorin and fluorouracil in patients with advanced carcinoma of the esophagus. Ann Oncol 16:1320-1325

7. Tanaka Y, Yoshida K, Sanada Y, Osada S, Yamaguchi K, Takahashi $\mathrm{T}$ (2010) Biweekly docetaxel, cisplatin, and 5-fluorouracil (DCF) chemotherapy for advanced esophageal squamous cell carcinoma: a phase I dose-escalation study. Cancer Chemother Pharmacol 66: $1159-1165$

8. Yamasaki M, Miyata H, Tanaka K, et al (2011) Multicenter phase I/ II study of docetaxel, cisplatin and fluorouracil combination chemotherapy in patients with advanced or recurrent squamous cell carcinoma of the esophagus. Oncologia 80:307-313

9. Tebbutt NC, Cummins MM, Sourjina T, et al. (2010) Randomized, non-comparative phase II study of weekly docetaxel with cisplatin and 5-fluorouracil or with capecitabine in oesophagogastric cancer: the AGITG ATTAX trial. Briti J Ca 102:475-481

10. Andreyev HJ, Norman AR, Oates J, et al (1998) Why do patients with weight loss have a worse outcome when undergoing chemotherapy for gastrointestinal malignancies? Eur J Cancer 34:503-509

11. Nishimura N, Nakano K, Ueda K, et al. (2012) Prospective evaluation of incidence and severity of oral mucositis induced by conventional chemotherapy in solid tumors and malignant lymphomas. Support Care Cancer 20:2053-2059

12. Common Terminology Criteria for Adverse Events (CTCAE), version 3.0 (2003) US Department of Health and Human Services, Washington.

13. Anderson PM, Schroeder G, Skubitz KM (1998) Oral glutamine reduces the duration and severity of stomatitis after cytotoxic cancer chemotherapy. Cancer 83(7):1433-1439

14. Huang EY, Stephen WL, Wang CJ, et al. (2000) Oral glutamine to alleviate radiation-induced oral mucositis: a pilot randomized trial. Int J Radiat Oncol Biol Phys 46(3):535-539

15. Aquino VM, Harvey AR, Garvin JH, et al. (2005) A double-blind randomized placebo-controlled study of oral glutamine in the prevention of mucositis in children undergoing hematopoietic stem cell transplantation: a pediatric blood and marrow transplant consortium study. Bone Marrow Transplant 36(7):611-616

16. Choi K, Lee SS, Oh SJ, et al. (2007) The effect of oral glutamine on 5-fluorouracil/leucovorin-induced mucositis/stomatitis assessed by intestinal permeability test. Clin Nutr 26:57-62

17. Cockerham MB, Weinberger BB, Lerchie SB (2000) Oral glutamine for the prevention of oral mucositis associated with highdose paclitaxel and melphalan for autologous bone marrow transplantation. Ann Pharmacother 34:300-303

18. Fukui T, Itoh Y, Yoshizawa K et al (2011) Elental prevented and reduced oral mucositis during chemotherapy in patients esophageal cancer. Gan to Kagaku Ryoho 38(13): 2597-2601.

19. Ogata Y, Takeuchi M, Ishibashi N, et al. (2012) Efficacy of elental on prevention for chemotherapy-induced oral mucositis in colorectal cancer patients. Gan To Kagaku Ryoho 39(4):583-587

20. Sonis ST (2004) The pathobiology of mucositis. Nat Rev Cancer 4: 277-284

21. Japan Esophageal Society (2009) Japanese classification of esophageal cancer, tenth edition: parts II and III. Esophagus 6:71-94

22. Oken MM, Creech RH, Tormey DC, et al (1982) Toxicity and response criteria of the Eastern Cooperative Oncology Group. Am J Clin Oncol 5:649-655

23. Tanaka Y, Yoshida K, Osada S, Yamaguchi K, Takahashi T (2011) Docetaxel, nedaplatin, and S-1 (DGS) chemotherapy for advanced esophageal squamous cell carcinoma: a phase I dose-escalation study. Anticancer Res 31:4589-4597 
24. Luk GD, Bayless TM, Baylin SB (1980) Diamine oxidase (histaminase). A circulating marker for rat intestinal mucosal maturation and integrity. J Clin Invest 66:66-70

25. Therasse P, Arbuck SG, Eisenhauer EA, et al. (2000) New guidelines to evaluate the response to treatment in solid tumors. European Organization for Research and Treatment of Cancer, National Cancer Institute of the United States, National Cancer Institute of Canada. J Natl Cancer Inst 92:205-216

26. Peterson DE, Jones JB, Petit 2nd RG (2007) Randomized, placebocontrolled trial of Saforis for prevention and treatment of oral mucositis in breast cancer patients receiving anthracycline-based chemotherapy. Cancer 109:322-331

27. May PE, Barber A, D'Olimpio JT, Hourihane A, Abumrad NN (2002) Reversal of cancer-related wasting using oral supplementation with a combination of beta-hydroxy-beta-methylbutyrate, arginine, and glutamine. Am J Surg 183:471-479

28. Yamamoto T, Nakahigashi M, Saniabadi AR, Iwata T, Maruyama Y, Umegae S, Matsumoto K (2007) Impacts of long-term enteral nutrition on clinical and endoscopic disease activities and mucosal cytokines during remission in patients with Crohn's disease: a prospective study. Inflamm Bowel Dis 13:1493-1501

29. Andou A, Hisamatsu T, Okamoto S, et al. (2009) Dietary histidine ameliorates murine colitis by inhibition of proinflammatory cytokine production from macrophages. Gastroenterology 136:564-574
30. Beaven MA, Shaff RE (1975) Study of the relationship of histaminase and diamine oxidase activities in various rat tissues and plasma by sensitive isotropic assay procedures. Biochem Pharmacol 24: 979-984

31. Namikawa T, Fukudome I, Kitagawa H, Okabayashi T, Kobayashi M, Hanazaki K (2012) Plasma diamine oxidase activity is a useful biomarker for evaluating gastrointestinal tract toxicities during chemotherapy with oral fluorouracil anti-cancer drugs in patients with gastric cancer. Oncology 82:147-152

32. Tsujikawa T, Uda K, Ihara T, Inoue T, Andoh A, Fujiyama Y, Bamba T (1999) Changes in serum diamine oxidase activity during chemotherapy in patients with hematological malignancies. Cancer Lett 147:195-198

33. Georgiannos SN, Weston PM, Goode AW (1993) Micronutrients in gastrointestinal cancer. Br J Cancer 68:1195-1198

34. Fijlstra M, Schierbeek H, Voortman G, Dorst KY, van Goudoever JB, Rings EH, Tissing WJ (2012) Continuous enteral administration can enable normal amino acid absorption in rats with methotrexate-induced gastrointestinal mucositis. J Nutr 142:19831990

35. Fijlstra M, Tissing WJ, Stellaard F, Verkade HJ, Rings EH (2013) Reduced absorption of long-chain fatty acids during methotrexate-induced gastrointestinal mucositis in the rat. Clin Nutr 32:452-459 